\title{
COMPLEMENTARY VARIATIONAL PRINCIPLES FOR A CLASS OF NONLINEAR BOUNDARY VALUE PROBLEMS
}

\author{
N. ANDERSON and A. M. ARTHURS
}

(Received 29 May 1978)

(Revised 7 August 1978)

\begin{abstract}
Complementary variational principles are presented for a class of nonlinear boundary value problems $S^{*} S \phi=g(\phi)$ in which $g$ is not necessarily monotone. The results are illustrated by two examples, accurate variational solutions being obtained in both cases.
\end{abstract}

\section{Introduction}

Complementary variational principles are known [1] for boundary value problems described by equations of the form

$$
T^{*} T \phi=f(\phi) \text { in } V, \quad \phi=0 \text { on } \partial V .
$$

Here $V$ is some region of $E^{n}$ with boundary $\partial V, T$ and $T^{*}$ form an adjoint pair of linear operators, and $f(\phi)$ is a monotonic decreasing function of $\phi$. We assume the existence of a solution $\phi$ of (1) and view it as an element in the real Hilbert space $H_{\phi}$ with inner product $\langle$,$\rangle . The operator T$ acts on elements in $H_{\phi}$ and sends them to a second real Hilbert space $H_{u}$ with inner product (, ). The adjoint $T^{*}$ of $T$ is defined by

$$
(v, T \psi)=\left\langle T^{*} v, \psi\right\rangle+(v, \sigma \psi)
$$

for all $v$ in $H_{u}$ and all $\psi$ in $H_{\phi}$, where $\sigma$ is a linear operator acting on functions on the boundary of $V$.

In this paper we investigate the possibility of extending these results to include boundary value problems in which $f(\phi)$ is not necessarily monotone decreasing. 
For instance, it may be monotone increasing, like $e^{\phi}$, or it may not be monotone at all, like $\sin \phi$. To consider problems such as these, we therefore look at a class of boundary value problems

$$
S * S \phi=g(\phi) \text { in } V, \quad \phi=0 \text { on } \partial V,
$$

where $S$ and $S^{*}$ form an adjoint pair of operators and where $g(\phi)$ is not necessarily monotonic decreasing. Our aim here is to rewrite (3) in a form corresponding to $(1)$, with $f(\phi)$ a monotonic decreasing function. This can be done for a certain class of problems.

First we shall suppose that $S^{*} S$ is a strictly positive operator, that is there exists a positive number $\lambda$ such that

$$
\left\langle\psi, S^{*} S \psi\right\rangle=(S \psi, S \psi) \geqslant \lambda\langle\psi, \psi\rangle
$$

for all non-zero $\psi$ in $H_{\phi}$. Then for some positive number $p$ we can write

$$
S^{*} S=T^{*} T+p, \quad p>0,
$$

for some positive self-adjoint operator $T^{*} T$, and equation (3) becomes

$$
T^{*} T \phi=f(\phi),
$$

with

$$
T^{*} T=S * S-p=L \text { say, }
$$

and

$$
f(\phi)=g(\phi)-p \phi .
$$

Now since $S^{*} S-p$ is positive, we must have

$$
p \leqslant \lambda_{0},
$$

where $\lambda_{0}$ is the lowest (positive) eigenvalue of the eigenproblem

$$
S * S \theta=\lambda \theta \text { in } V, \quad \theta=0 \text { on } \partial V .
$$

In addition we want $f(\phi)$ to be monotone decreasing and this means that

$$
\frac{g\left(\phi_{i}\right)-g\left(\phi_{j}\right)}{\phi_{i}-\phi_{j}} \leqslant p
$$

for all $\phi_{i}$ and $\phi_{j}$ in $H_{\phi}$. If $g(\phi)$ is differentiable this becomes

$$
g^{\prime}(\psi) \leqslant p, \text { for all } \psi \text { in } H_{\phi} \text {. }
$$

Combining (9) and (12) we therefore find that $p$ must satisfy the conditions

$$
g^{\prime}(\psi) \leqslant p \leqslant \lambda_{0} \text { for all } \psi \text { in } H_{\phi} .
$$


We shall assume that such a number $p$ can be found.

To derive complementary variational principles associated with (3), rewritten as (6), we wish to use a canonical approach. We therefore require, at least in theory, the operators $T$ and $T^{*}$, and if we assume that we can write

$$
T=S+q,
$$

then

$$
T^{*}=S^{*}+q,
$$

where $q$ is some function as yet unknown. Then (7) requires that

$$
S^{*}(q \psi)+q S \psi+q^{2} \psi+p \psi=0 \text { for all } \psi \text { in } H_{\phi} .
$$

This is of the form

$$
A(q) \psi=0,
$$

and so we require a $q$ which satisfies

$$
A(q)=0
$$

at all points of the space $V$. As we shall see later, beyond its existence, knowledge of $q$ is not needed in practice for the class of problems under consideration.

\section{Complementary principles}

In Section 1 the boundary value problem (3) has been rewritten in the form

$$
T^{*} T \phi=f(\phi) \text { in } V, \quad \phi=0 \text { on } \partial V .
$$

We now derive the associated complementary variational principles.

We write (18) in canonical form

$$
\begin{array}{ll}
\Omega_{1}: & T \phi=u=W_{u}, \quad \phi=0 \text { on } \partial V, \\
\Omega_{2}: & T^{*} u=f(\phi)=W_{\phi} \text { in } V,
\end{array}
$$

where subscripts on the Hamiltonian $W$ denote abstract derivatives. A suitable $W$ is given by

$$
W(u, \phi)=\frac{1}{2}(u, u)+F(\phi),
$$

where

$$
F(\phi)=\int^{\phi}\langle f(\psi), d \psi\rangle
$$


Equations (19) and (20) are the Euler-Hamilton equations associated with the action functional

$$
\begin{aligned}
I(u, \phi) & =(u, T \phi)-W(u, \phi)-(u, \sigma \phi) \\
& =\left\langle T^{*} u, \phi\right\rangle-W(u, \phi) .
\end{aligned}
$$

Using (21) we see that

$$
\begin{aligned}
I(u, \phi) & =(u, T \phi)-\frac{1}{2}(u, u)-F(\phi)-(u, \sigma \phi) \\
& =\left\langle T^{*} u, \phi\right\rangle-\frac{1}{2}(u, u)-F(\phi) .
\end{aligned}
$$

The action $I$ is stationary at the solution $(u, \phi)$ of equations (19) and (20).

Now we define a pair of dual functionals as follows:

$$
\begin{aligned}
J\left(\phi_{1}\right) & =I\left(u_{1}, \phi_{1}\right) \quad \text { via }(24 a), \text { with }\left(u_{1}, \phi_{1}\right) \text { in } \Omega_{1} \\
& =\frac{1}{2}\left(T \phi_{1}, T \phi_{1}\right)-F\left(\phi_{1}\right) \\
& =\frac{1}{2}\left\langle\phi_{1}, L \phi_{1}\right\rangle-F\left(\phi_{1}\right), \text { with } \phi_{1}=0 \text { on } \partial V,
\end{aligned}
$$

and

$$
\begin{aligned}
K\left(u_{2}\right) & =I\left(u_{2}, \phi_{2}\right) \quad \text { via }(24 \mathrm{~b}), \text { with }\left(u_{2}, \phi_{2}\right) \text { in } \Omega_{2} \\
& =\left\langle T^{*} u_{2}, f^{-1}\left(T^{*} u_{2}\right)\right\rangle-\frac{1}{2}\left(u_{2}, u_{2}\right)-F\left[f^{-1}\left(T^{*} u_{2}\right)\right]
\end{aligned}
$$

If we take

$$
u_{2}=T \psi_{2}, \quad \psi_{2}=0 \text { on } \partial V
$$

we have

$$
K\left(T \psi_{2}\right)=\left\langle L \psi_{2}, f^{-1}\left(L \psi_{2}\right)\right\rangle-\frac{1}{2}\left\langle\psi_{2}, L \psi_{2}\right\rangle-F\left[f^{-1}\left(L \psi_{2}\right)\right]
$$

Here

$$
L=T^{*} T=S^{*} S-p,
$$

and

$$
f(\psi)=g(\psi)-p \psi
$$

Since

$$
f^{\prime}(\psi) \leqslant 0 \text { for all } \psi,
$$

we have the complementary extremum principles [cf. 2]

$$
K\left(T \psi_{2}\right) \leqslant K(T \phi)=J(\phi) \leqslant J\left(\phi_{1}\right)
$$


equality holding when $\phi_{1}$ and $\psi_{2}$ are equal to the solution $\phi$. With $J$ in (25) and $K$ in (28), we note that the function $q$ of (14) is not required explicitly.

There is an alternative form for $J\left(\phi_{1}\right)$ which we give here. By (25)

$$
\begin{aligned}
J\left(\phi_{1}\right) & =\frac{1}{2}\left\langle\phi_{1}, L \phi_{1}\right\rangle-F\left(\phi_{1}\right) \\
& =\frac{1}{2}\left\langle\phi_{1},\left(S^{*} S-p\right) \phi_{1}\right\rangle-\int^{\phi_{1}}\left\langle g(\psi)-p \psi, a^{\prime} \psi\right\rangle \\
& =\frac{1}{2}\left\langle\phi_{1}, S^{*} S \phi_{1}\right\rangle-G\left(\phi_{1}\right) \\
& =\frac{1}{2}\left(S \phi_{1}, S \phi_{1}\right)-G\left(\phi_{1}\right),
\end{aligned}
$$

where

$$
G\left(\phi_{1}\right)=\int^{\phi_{1}}\langle g(\psi), d \psi\rangle
$$

Thus we have a formula (33) for $J\left(\phi_{1}\right)$ in terms of the original operators and functions of equation (3). In this form the number $p$ drops out and the minimum principle for $J$ holds provided that

$$
g^{\prime}(\psi) \leqslant \lambda_{0} \text { for all } \psi,
$$

which of course is consistent with equation (13).

\section{Example 1}

To illustrate these ideas we first consider the nonlinear two-point boundary value problem described by the equations

$$
\frac{d^{2} \phi}{d x^{2}}=-e^{\phi}, \quad 0<x<1,
$$

and

$$
\phi(0)=\phi(1)=0 .
$$

It is known [5] that there is a non-negative solution $\phi$ such that

$$
0 \leqslant \phi<0.142 \text {. }
$$

This is an example of our class of problems in (3) with

$$
\begin{gathered}
S=\frac{d}{d x}, \quad S^{*}=-\frac{d}{d x}, \\
g(\phi)=e^{\phi},
\end{gathered}
$$


and

$$
\langle\phi, \psi\rangle=\int_{0}^{1} \phi \psi d x, \quad(u, v)=\int_{0}^{1} u v d x
$$

Here

$$
g^{\prime}(\psi)=e^{\psi} \geqslant 0
$$

and so $g(\phi)$ is monotone increasing. To reformulate the problem as in Section 1 we need to find a positive number $p$ such that

$$
g^{\prime}(\psi) \leqslant p \leqslant \lambda_{0} \text { for all } \psi
$$

If we restrict all admissible functions $\psi$ to the range

$$
0 \leqslant \psi<0.142 \text {, }
$$

this means that

$$
\exp (0.142) \leqslant p \leqslant \pi^{2}
$$

which provides a choice of possible $p$ values.

For this example we find that the function $q$ of (16) and (17) must satisfy

$$
-q^{\prime}+q^{2}+p=0
$$

and this has the general solution

$$
q=\sqrt{ }(p) \tan \{\sqrt{ }(p)(x+c)\}
$$

where $c$ is a constant. With $p$ in the range specified by (45), and $0<x<1$, the existence of $q$ over the whole range is assured by the choice $c=-\frac{1}{2}$. We can therefore use the results of Section 2 with

$$
L=-\frac{d^{2}}{d x^{2}}-p
$$

and

$$
f(\psi)=e^{\psi}-p \psi
$$

By (25) and (28), the dual functionals $J$ and $K$ are

$$
\begin{aligned}
J\left(\phi_{1}\right) & =\int_{0}^{1}\left\{\frac{1}{2} \phi_{1} L \phi_{1}-e^{\phi_{1}}+\frac{1}{2} p \phi_{1}^{2}\right\} d x \\
& =\int_{0}^{1}\left\{\frac{1}{2}\left(\phi_{1}^{\prime}\right)^{2}-e^{\phi_{1}}\right\} d x, \quad \phi_{1}(0)=\phi_{1}(1)=0,
\end{aligned}
$$


and

Since

$$
\begin{array}{r}
K\left(T \psi_{2}\right)=\int_{0}^{1}\left\{\left(L \psi_{2}\right) f^{-1}\left(L \psi_{2}\right)-\frac{1}{2} \psi_{2} L \psi_{2}-\exp \left[f^{-1}\left(L \psi_{2}\right)\right]\right\} d x \\
\psi_{2}(0)=\psi_{2}(1)=0 .
\end{array}
$$

$$
f^{\prime}(\psi)=e^{\psi}-p<0
$$

for all functions $\psi$ satisfying (44), we see that (31) is satisfied, and the global complementary principles

$$
K\left(T \psi_{2}\right) \leqslant K(T \phi)=J(\phi) \leqslant J\left(\phi_{1}\right)
$$

hold. The minimum principle for $J$ was given previously by Arthurs and Winthrop [4], but the maximum principle for $K$ appears to be new.

We can use (52) to obtain an approximation to the exact function $\phi$. Taking $p=\exp (0.142)$ we have performed calculations with the trial functions

$$
\left.\begin{array}{l}
\phi_{1}=\sum_{n=1}^{3} a_{n}\left(x-x^{2}\right)^{n}, \\
\psi_{2}=\sum_{n=1}^{3} b_{n}\left(x-x^{2}\right)^{n},
\end{array}\right\}
$$

where the parameters $a_{n}$ and $b_{n}$ were determined by optimizing $J$ and $K$.

The results are

$$
\begin{aligned}
a_{1}=0.54920013, & b_{1}=0.55020013, \\
a_{2}=0.05310009, & b_{2}=0.05300009, \\
a_{3}=-0.00498991, & b_{3}=-0.00301991, \\
J=-1.0465168, & K=-1.0465168 .
\end{aligned}
$$

Since $J-K$ provides a measure of the mean square error in the function $\phi_{1}$ [see 3], we conclude that $\phi_{1}$ is a very good approximate solution of the problem in (36) and (37). The estimate (slightly corrected) given in [4] shows that

$$
\left|\phi_{1}-\phi\right|<1.7 \times 10^{-4} \text {. }
$$

\section{Example 2}

Our second example concerns the nonlinear two-point boundary value problem

$$
\frac{d^{2} y}{d x^{2}}+\sin y(x)=0, \quad 0<x<3,
$$


with

$$
y(0)=0, \quad y(3)=B>0 \text {. }
$$

This has been studied numerically by Bailey et al. [5] and they find that iteration methods provide relatively slow convergence to the unique solution $y$.

Since the boundary conditions in (56) are not homogeneous we shall make them so by setting

$$
y(x)=\phi(x)+\frac{1}{3} B x
$$

which gives the new problem

$$
\frac{d^{2} \phi}{d x^{2}}+\sin \left(\phi+\frac{1}{3} B x\right)=0, \quad 0<x<3
$$

with

$$
\phi(0)=\phi(3)=0 \text {. }
$$

Equations (58) and (59) provide an example of our class of problems in (3) with

$$
\begin{aligned}
& S=\frac{d}{d x}, \quad S^{*}=-\frac{d}{d x}, \\
& g(\phi)=\sin \left(\phi+\frac{1}{3} B x\right),
\end{aligned}
$$

and

$$
\langle\phi, \psi\rangle=\int_{0}^{3} \phi \psi d x, \quad(u, v)=\int_{0}^{3} u v d x .
$$

Here we see that $g(\phi)$ is not monotone.

To reformulate the problem as in Section 1 we need to find a positive number $p$ such that

$$
g^{\prime}(\psi) \leqslant p \leqslant \lambda_{0} \text { for all } \psi
$$

For this example, (63) is satisfied by choosing $p$ in the range

$$
1 \leqslant p \leqslant \frac{1}{9} \pi^{2} .
$$

The function $q$ in the decomposition (14) again satisfies (46) and is given over the whole range by (47) with $c$ chosen to be $c=-\frac{3}{2}$. We can therefore use the results of Section 2 with

$$
L=-\frac{d^{2}}{d x^{2}}-p
$$

and

$$
f(\psi)=\sin \left(\psi+\frac{1}{8} B x\right)-p \psi
$$


By (25) and (28) the dual functionals $J$ and $K$ are

$$
\begin{aligned}
J\left(\phi_{1}\right) & =\int_{0}^{3}\left\{\frac{1}{2} \phi_{1} L \phi_{1}+\cos \left(\phi_{1}+\frac{1}{3} B x\right)+\frac{1}{2} p \phi_{1}^{2}\right\} d x \\
& =\int_{0}^{3}\left\{\frac{1}{2}\left(\phi_{1}^{\prime}\right)^{2}+\cos \left(\phi_{1}+\frac{1}{3} B x\right)\right\} d x, \quad \phi_{1}(0)=\phi_{1}(3)=0,
\end{aligned}
$$

and

$$
\begin{gathered}
K\left(T \psi_{2}\right)=\int_{0}^{3}\left\{\left(L \psi_{2}\right) f^{-1}\left(L \psi_{2}\right)-\frac{1}{2} \psi_{2} L \psi_{2}+\frac{1}{2} p\left[f^{-1}\left(L \psi_{2}\right)\right]^{2}+\cos \left[f^{-1}\left(L \psi_{2}\right)+\frac{B x}{3}\right]\right\} d x \\
\psi_{2}(0)=\psi_{2}(3)=0 .
\end{gathered}
$$

Since

$$
f^{\prime}(\psi)=\cos \left(\psi+\frac{1}{3} B x\right)-p \leqslant 0
$$

for values of $p$ in the range (64), we see that (31) is satisfied and the global complementary principles

$$
K\left(T \psi_{2}\right) \leqslant K(T \phi)=J(\phi) \leqslant J\left(\phi_{1}\right)
$$

hold. These principles appear to be new.

To obtain an approximate solution of (58) and (59), and hence of (55) and (56), we have performed calculations with the trial functions

$$
\phi_{1}=\sum_{n=1}^{7} a_{n}\left\{\left(\frac{1}{3} x\right)^{n+1}-\frac{1}{3} x\right\}
$$

and

$$
\psi_{2}=\sum_{n=1}^{3} b_{n}\{x(3-x)\}^{n}
$$

To avoid difficulties with $f^{-1}$ in (68) we took

$$
p=\frac{1}{9} \pi^{2},
$$

and for comparison with the results in [5] we chose

$$
B=2.7 .
$$


The parameters $a_{n}$ and $b_{n}$ were found by optimizing $J$ and $K$, and the results are

$$
\begin{array}{lll}
a_{1}=-2.445, & a_{5}=-0.423, & b_{1}=0.320, \\
a_{2}=-2.937, & a_{6}=-0.078, & b_{2}=0.0356, \\
a_{3}=1.322, & a_{7}=-0.145, & b_{3}=-0.0042, \\
a_{4}=1.285, & J=-0.2546, & K=-0.2822 .
\end{array}
$$

By (57), our variational solution of the original problem in (55) and (56) is

$$
y_{1}=\phi_{1}+\frac{1}{3} B x
$$

The variational bounds in (75) indicate that $y_{1}$ is quite a good approximate solution and to check this we have also obtained a numerical solution. Table 1 provides a comparison between these two solutions which are seen to be in very close agreement.

TABLE 1

Comparison of variational and numerical solutions in example 2

\begin{tabular}{llc}
\hline \multicolumn{1}{c}{$x$} & \multicolumn{1}{c}{$y_{1}$} & $\begin{array}{c}y \\
\text { (numerical) }\end{array}$ \\
\hline 0 & 0 & 0 \\
0.5 & 0.938 & 0.938 \\
0.75 & 1.335 & $1.343 \dagger$ \\
1.0 & 1.678 & 1.688 \\
1.5 & 2.193 & $2.197 \dagger$ \\
2.0 & 2.502 & 2.505 \\
2.25 & 2.595 & $2.599 \dagger$ \\
2.5 & 2.657 & 2.661 \\
3.0 & 2.700 & 2.700 \\
\hline & \\
\hline &
\end{tabular}

\section{References}

[1] A. M. Arthurs, "Extremum principles for a class of boundary value problems", Proc. Camb. Phil. Soc. 65 (1969), 803-806.

[2] A. M. Arthurs, Complementary variational principles (Clarendon Press, Oxford, 1970).

[3] A. M. Arthurs, "Dual extremum principles and error bounds for a class of boundary value problems", J. Math. Anal. Appl. 41 (1973), 781-795. 
[4] A. M. Arthurs and D. Winthrop, "Variational solution and error bound for a nonlinear boundary value problem in heat diffusion", Nuovo Cimento Lett. 7 (1973), 150-152.

[5] P. B. Bailey, L. F. Shampine and P. E. Waltman, Nonlinear two-point boundary value problems (Academic Press, New York, 1968).

\section{Department of Mathematics}

University of York

York, YO1 5DD

England 